\title{
Special Issue: Applications of Green-Synthesized Nanoparticles in Pharmacology, Parasitology and Entomology
}

\author{
Giovanni Benelli ${ }^{1}$ - Charles M. Lukehart ${ }^{2}$
}

Published online: 17 January 2017

(C) Springer Science+Business Media New York 2017

This Special Issue of Journal of Cluster Science contains the first two Commentary articles published in this journal along with a collection of 38 invited or contributed original papers reporting applications of green-synthesized nanoparticles to the fields of pharmacology, parasitology and entomology. Nanobiotechnologies have the potential to revolutionize a wide array of clinical and environmental applications, including drug delivery, diagnostics, imaging, sensing, gene delivery, artificial implants, tissue engineering, parasitology, and pest management. Nanoparticulate materials are of particular interest for many such applications due to their unusual optical, chemical and photo-electrochemical properties. Biofabrication ("green" preparation methods) of nanoparticulate materials exploits microbial and botanical-based, single-step synthesis strategies that do not require high pressure, intensive energy consumption, elevated temperatures, or use of highly toxic chemicals more typical of chemical and physical methods. Although utilizing botanical extracts as chemical reagents in nanoparticle formation complicates identification of reactive species, in situ surface-passification usually occurs to give biocompatible nanoparticle dispersions ideally suitable for clinical, biological, or environmental testing.

A Commentary (Benelli, Pavela, et al.) leads off this Special Issue on the topic of using plant products for nanoparticle syntheses relevant to pest control, an outcome closely related to human health. Because proper data analysis has general importance to quantifying effects of bionanoscience applications, the second Commentary (Benelli) provides readers with an overview of this relevant topic.

Giovanni Benelli

benelli.giovanni@gmail.com

Charles M. Lukehart

chuck.lukehart@vanderbilt.edu

1 Department of Agriculture, Food and Environment, University of Pisa, 56124 Pisa, Italy

2 Department of Chemistry, Vanderbilt University, Nashville, TN 37235, USA 
In articles that follow, green syntheses of $\mathrm{Ag}, \mathrm{Au}, \mathrm{MoO}_{3}, \mathrm{Ag}_{2} \mathrm{~S}, \mathrm{ZnO}, \boldsymbol{\alpha}-\mathrm{Fe}_{2} \mathrm{O}_{3}$, $\mathrm{Cu}, \mathrm{Fe}$, graphene, $\mathrm{Pd}, \mathrm{FeO}, \mathrm{Se}, \mathrm{CuS}, \mathrm{SiO}_{2}$ or $\mathrm{TiO}_{2}$ nanoparticles are reported for a wide variety of applications related to pharmacology, parasitology and entomology. Biomedical applications investigated include (1) antibacterial and antifungal activity, even on multi-drug resistant organisms (Skladanowski, et al.; Anbazhagan, et al.; Dighore, et al.; Sekar, et al.; Balasubramani, et al.; Elemike, et al.; Mukherjee; Bhakyaraj, et al.; Agale, et al.; Jinu, et al.; Prasannaraj, et al.; Venkatachalam, et al.; Prasannaraj, et al.), (2) antiparasitic activity on Plasmodium falciparum strains resistant to chloroquine (Theerthagiri, et al.), (3) antiviral activity against HIV, due to inhibition of HIV-1 reverse transcriptase (Kumar, Singaravelu, Ajithkumar, et al.), and dengue virus (serotype DEN-2) growth inhibition blocking the production of the DEN-2 envelope (E) protein (Murugan, Dinesh, Paulpandi, et al.), (4) toxicity on different cancer cell lines (Pandurangan, et al.; Patil, et al.; Vijayashree, et al.; Ashokan, et al.; Nagajyothi, et al.; Balasubramani, et al.; Devanesan, et al.; Gopinath, et al.), (5) lower toxicity on mammalian cell lines (e.g. Vero cells), when compared to chemically synthesized nanoparticles (Anu, et al.; Kumar, Singaravelu, Murugan, et al.).

Applications relevant to pest management science, with special reference to the eco-friendly control of mosquito vectors of public health importance, include contributions by Govindarajan, et al.; Vincent, et al.; Anbazhagan, et al.; Azeez, et al.; Azarudeen, Govindarajan, Amsath, et al.; Ashokan, et al.; Benelli and Govindarajan; Govindarajan and Benelli; Azarudeen, Govindarajan, Marzouq, et al.; Murugan, Nataraj, et al.; Subramaniam, et al.; Bhakyaraj, et al.; Kurugan, et al.; Mahyoub, et al., Theerthagiri, et al.; and, Benelli, Govindarajan, Kadaikunnan, et al. It is worthy to note that some of these reports focus on control of Zika virus vectors (Azarudeen, Govindarajan, Amsath, et al.; Azarudeen, Govindarajan, Marzouq, et al.; Mahyoub, et al.), which recently gained high research attention worldwide, due to the recent Zika virus outbreaks in the Americas and in the Pacific. Notably, a number of studies reveal lower toxicity rates of metal and graphene nanoparticles on non-target aquatic organisms (e.g. fishes, tadpoles and water bugs) when compared to targeted mosquito vectors (Murugan, Nataraj, Jaganathan, et al.). However, other reports highlight some caveats concerning employment of biofabricated nanoparticles in aquatic environments, due to accumulation of metal nanoparticles in tissues of micro-crustaceans and fishes (Ramachandran, et al.).

More specialized studies include use of $\mathrm{Ag}_{2} \mathrm{~S}$ quantum dots for in vivo imaging along with evaluation of the toxicity and hemolytic activity of $\mathrm{Ag}_{2} \mathrm{~S}$ nanoparticles toward human red blood cells (Javidi, et al.) and promotion of cell division and pollen germination in plant cells by Au nanoparticles (Alharbi, et al.). It is hoped that readers of this Special Issue will gain insight and appreciation of the breadth of applications realized through the use of green-synthesized nanoparticles. 